\title{
Tivozanib in the treatment of renal cell carcinoma
}

This article was published in the following Dove Press journal:

Biologics:Targets and Therapy

10 June 2013

Number of times this article has been viewed

\section{Mehmet Hepgur \\ Sarmad Sadeghi \\ Tanya B Dorff \\ David I Quinn}

Division of Medical Oncology, University of Southern California Norris Comprehensive Cancer Center, Keck School of Medicine, Los Angeles, CA, USA
Correspondence: David I Quinn Division of Medical Oncology, University of Southern California Norris Comprehensive Cancer Center, Keck School of Medicine, I44I Eastlake Avenue, Suite 3440, Los Angeles, CA 90033, USA

Tel +l 3238653956

Fax + | 323865006 |

Email diquinn@med.usc.edu
Abstract: Renal cell carcinoma (RCC) is an aggressive malignancy compared to other urological malignancies and has been associated with poor responses to conventional cytotoxic chemotherapy. Interferon- $\alpha$ and interleukin- 2 were previously utilized in a limited number of patients with good performance status due to toxicity and safety issues. Over the last decade, through advances in the understanding of the biology and pathology of RCC, the important role of vascular endothelial growth factor (VEGF) in RCC has been identified. Data from randomized trials have led to the approval of first-generation tyrosine kinase inhibitors (TKIs) sorafenib, sunitinib, and pazopanib; however, these agents inhibit a wide variety of kinase targets and are associated with a range of adverse effects. More recently, a new generation TKI, axitinib, has been approved by the US Food and Drug Administration. Tivozanib is a novel TKI, which is a potent inhibitor of VEGF-1, VEGF-2, VEGF-3, c-kit, and PDGR kinases, with a more restricted target spectrum. Phase II and III studies have demonstrated significant activity and a favorable safety profile as an initial targeted treatment for advanced RCC. This review examines the emerging data with tivozanib for the treatment of advanced RCC. Preclinical investigations as well as Phase I, II, and III data are examined; data on the comparative benefits of tivozanib are reviewed. Finally, we discuss the future potential of tivozanib in combination, biomarkers associated with tivozanib response, and acquisition of resistance and nonkidney cancer indications.

Keywords: targeted therapy, renal cell cancer, tyrosine kinase inhibitor, tivozanib

\section{Introduction}

Renal cell carcinoma (RCC) accounts for approximately 3\% of adult malignancies and $90 \%-95 \%$ of all malignant kidney tumors. On January 1, 2009, in the United States, there were approximately 320,182 men and women alive who had a history of cancer of the kidney and renal pelvis. It is estimated that 64,770 men and women (40,250 men and 24,520 women) were diagnosed with, and 13,570 men and women died of cancer of the kidney and renal pelvis in 2012. ${ }^{1,2}$

$\mathrm{RCC}$ is an aggressive malignancy compared to other urological malignancies; ${ }^{3}$ $20 \%-30 \%$ of patients present with metastatic disease due to the insidious nature of the disease and absence of a screening test. ${ }^{4}$ Approximately $25 \%$ of patients with early-stage disease at diagnosis relapse after nephrectomy. ${ }^{5}$ Patients with advanced disease have a 5-year survival rate of $10 \%$, compared to $85 \%$ for patients with localized RCC. Therefore, advanced RCC has been associated with a poor prognosis and poor responses to conventional cytotoxic chemotherapy. Immunotherapy with high-dose interleukin (IL)-2 or interferon-alpha (INF- $\alpha$ ) has been used as therapy in select patients with good performance status. The response rate with immunotherapy is relatively 
low $(5 \%-30 \%)$, and toxicity and safety issues often limit their use..$^{6-8}$ The clinical efficacy of INF- $\alpha$ is limited; a metaanalysis reviewed data from 463 patients with advanced RCC who were administered INF- $\alpha$ as first-line systemic therapy and suggested that treatment with INF- $\alpha$ provides a median progression-free survival (PFS) of 4.7 months and a median overall survival (OS) of 13 months. ${ }^{9}$ In the cytokine era, this result was proposed as a benchmark for the future targeted therapies. ${ }^{9,10}$

Advances in the understanding of the biology and pathology of RCC identified the important role of vascular endothelial growth factor (VEGF). This has led to development of numerous targeted agents, which have established a new benchmark for PFS and OS in Phase III trials compared with INF- $\alpha .{ }^{11-13}$ Data from randomized trials have led to the approval of seven agents over the last 5 years for advanced RCCs in first- or second-line treatment in metastatic RCC: sunitinib, sorafenib, bevacizumab (with or without INF- $\alpha$ ), temsirolimus, everolimus, pazopanib, and axitinib. ${ }^{14}$ Unfortunately, the existing treatment options have several restrictions: durable responses are rare; some cases show no response; the median prolongation in PFS is generally measured in months; and in some responding patients, toxicities can make long-term treatment difficult or necessitate dose reductions and interruptions. Because of these issues, additional therapies are still needed. Tivozanib (AV-951) is a novel agent that is a potent inhibitor of VEGF-1, VEGF-2, VEGF-3, c-kit, and PDGR kinases. Phase II and III studies have demonstrated significant activity and favorable safety profile as initial targeted treatment for advanced RCC. ${ }^{15,16}$

\section{Molecular biology of RCC}

The biology of hereditary renal neoplasms has led to a better understanding of the molecular basis of clear cell RCC. The Von Hippel-Lindau (VHL) tumor suppressor gene, located on chromosome $3 \mathrm{p} 25$, is mutated in patients with hereditary VHL syndrome, inducing cysts and tumors in the central nervous system and abdomen such as hemangioblastoma and pheochromocytoma. This condition also includes a predisposition to the development of clear cell RCC. ${ }^{17}$ Interestingly, VHL gene inactivation has also been observed in $84 \%$ to $98 \%$ of sporadic RCCs based upon specific mutations or epigenetic gene silencing. ${ }^{18-24}$ Thus, VHL abnormalities are key in the pathogenesis of clear cell RCC.

VHL protein regulates normal cellular responses to hypoxia by ubiquitin-mediated hypoxia-inducible factor 1 alpha (HIF1alpha). Using normal oxygen levels, oxygen content in the blood controls the formation of VHL protein complexes, by targeting HIF1alpha for deprivation by proteasomes. Low oxygen conditions lead to HIF1alpha accumulation and bind to HIF1beta, followed by formation of a complex that transcriptionally activates genes. Similarly, mutation or inactivation of the VHL protein disrupts the ability to degrade HIF1alpha, causing excessive accumulation even under normal oxygen conditions. This abnormal response activates the hypoxia response pathway and galvanizes numerous HIF-responsive genes, which leads to overproduction of proangiogenic factors, including growth factors such as VEGF, platelet-derived growth factor (PDGF), and transforming growth factor alpha/beta. HIF accumulation also takes place with activation of the mammalian target of rapamycin (mTOR) through cellular stimuli and the phosphoinositide 3-kinase/Akt (protein kinase) pathway. ${ }^{25}$ Activated mTOR also leads to a cascade of events such as phosphorylation of $4 \mathrm{E}$ binding protein-1, leading to eukaryotic initiation factor-4 subunit E enhancing the translation of mRNAs that encode cell-cycle regulators such as cyclin D1 and c-myc. ${ }^{26,27}$ Sulzbacher et $\mathrm{al}^{28}$ documented PDGF expression in a large number of RCCs. PDGF receptor expression was correlated with higher in highly malignant tumors and adverse outcomes for patients with RCC. These pathways are involved in tumor proliferation, ${ }^{29-32}$ as well as in promoting tumor-associated angiogenesis. ${ }^{33}$

VEGF primarily affects vascular endothelial cells by binding to and activating three structurally similar receptor tyrosine kinases: ${ }^{30}$ VEGFR1 (also known as FLT1), VEGFR2 (also known as KDR), and VEGFR3 (also known as FLT4). Each VEGF ligand has a specific binding affinity for each of these tyrosine kinase receptors, which contributes to their diversity. Therefore, binding of each VEGFR tyrosine kinase activates a distinct downstream pathway that leads to diverse effects that leads to tumor proliferation and metastasis by mediating numerous changes within the tumor vasculature and promoting tumor angiogenesis. ${ }^{18,33}$ Tumor angiogenesis is enhanced through increasing blood flow by promoting endothelial cell proliferation and survival; enhancing migration of endothelial cells; and increased permeability of existing cellular transport channels, developing an environment for endothelial cell migration through boosting chemotaxis of bone marrow derived endothelial cell precursors. ${ }^{18,34-36}$

\section{Inhibition of VEGF in renal cell carcinoma}

The inhibition of VEGF signaling may affect tumor growth through several mechanisms, such as antiangiogenic effects, 
induction of endothelial cell apoptosis, and blockade of migration of hematopoietic and endothelial progenitor cells. The angiogenesis in RCC, in both the primary and metastatic sites of disease, is presumed to be highly VEGF-dependent due to the high-frequency inactivation of the VHL tumor suppressor gene. Therefore, VEGF has been an important target in development of therapeutic agents in RCC.

RCC is one of the most immunogenic tumors. ${ }^{5,37}$ Programmed cell death 1 is one of these immunogenic mechanisms. BMS-936558, a monoclonal antibody directed at the programmed cell death $1 \mathrm{~T}$-cell coinhibitory receptor, has been investigated in a Phase I study. In the RCC cohort, nine of 33 patients had an objective response. ${ }^{38}$ Another monoclonal antibody, BMS-936559, targeting programmed cell death 1 ligand protein, is also being studied (ClinicalTrials.gov identifier: NCT01354431). ${ }^{39}$ In the tumor microenvironment, VEGF weakens the host immune response by reducing dendritic cell differentiation and increasing secretion of immunosuppressive cytokines such as IL-10 and transforming growth factor beta. ${ }^{34}$ Therefore, VEGF-inhibitors may also take part in modulation of the antitumor immunity. ${ }^{40}$

VEGF-targeted agents include bevacizumab, a humanized monoclonal antibody that inhibits VEGF A, and receptor tyrosine kinase inhibitors (TKIs) such as sorafenib, sunitinib, pazopanib, and axitinib. ${ }^{29,41}$ These agents target the VEGF receptors, as do additional TKIs in ongoing clinical development. Amongst the three receptors for VEGF, VEGFR-2 on endothelial cells appears to be the key target. ${ }^{42}$ This model initiated the development of novel agents with enhanced targeting of VEGFR2, such as tivozanib.

\section{Preclinical investigations and tivozanib pharmacology}

Tivozanib is a quinoline-urea derivative, a small molecule recently developed as a third-generation VEGF receptor TKI. ${ }^{43}$ In preclinical studies, oral administration of tivozanib to athymic rats decreased the microvessel density within tumor xenografts and reduced VEGFR-2 phosphorylation levels in tumor endothelium, triggering inhibition of angiogenesis and vascular permeability. It also demonstrated antitumor activity in several human tumor xenografts, including lung, breast, colon, ovarian, pancreas, and prostate cancer. Preclinical activity for tivozanib in colorectal cancer was demonstrated by inhibition of progression in the peritoneal cavity in a colon cancer model with reduced tumor angiogenesis, ascites formation, and tumor spread, thereby prolonging survival. ${ }^{44}$
The activity of tivozanib has been assessed against several receptor tyrosine kinases using either cell-free kinase assays or the inhibition of ligand-induced phosphorylation of growth factor receptors. Tivozanib is a potent and selective VEGFR TKI with the highest potency for VEGFR-2, with similar levels for VEGFR-1 and VEGFR-3 (Table 1). Tivozanib showed relatively less inhibitory activity of c-kit, PDGF receptor (PDGFR)-b, Flt-3, fibroblast growth factor receptor-1, and c-met. Tivozanib has virtually no activity for EGFR and insulin-like growth factor-1. The specific potency of tivozanib on VEGFR-2 provides a theoretical distinction compared to earlier drugs used in RCC treatment such as sorafenib, sunitinib, and pazopanib.

Multitargeted TKIs may have a theoretical advantage of increased antitumor activity and delaying resistance; however, off-target effects may contribute to adverse events. Hand-foot syndrome, skin rash, fatigue, stomatitis, diarrhea, pigmentation changes, myelosuppression, and thyroid dysfunction are commonly associated with multitargeted TKI treatments. ${ }^{10,45}$ Low potency of first-generation TKIs requires administration of higher doses to obtain optimal VEGFR inhibition. This usually leads to off-target toxicities at therapeutic doses. Compared with other US Food and Drug Administration-approved small-molecule TKIs, tivozanib is relatively specific for VEGFR-1, VEGFR-2, and VEGFR3, although it still exhibits PDGFR-b inhibition. Although IC50 determination can be variable, depending on the cell system used, the IC50 concentrations for tivozanib inhibiting VEGFRs are the lowest in this group of agents (Table 1).

\section{Phase I studies}

Prior pharmacokinetic analysis in healthy volunteers demonstrated good oral bioavailability, slow absorption, predominantly fecal excretion, and long half-life. In this study, the half-life was 4.7 days, suggesting suitability for once-daily dosing. ${ }^{46}$ Concentration-time profiles from the

Table I Receptor tyrosine kinase inhibitory activity at $50 \%$ of US Food and Drug Administration-approved TKIs in cell-free kinase assay (IC50)

\begin{tabular}{llllll}
\hline TKI & Tivozanib & Axitinib & Sunitinib & Pazopanib & Sorafenib \\
\hline VEGFR-I & 30 & ND & 21 & 7 & 9 \\
VEGFR-2 & 6.5 & 0.2 & 34 & 15 & 28 \\
VEGFR-3 & 15 & ND & 3 & 2 & 7 \\
PDGFR-B & 49 & 1.6 & 75 & 215 & 1129 \\
c-Kit & 78 & 1.7 & 40 & 48 & 1862 \\
FGFR-I & 530 & ND & 437 & 80 & 64 \\
\hline
\end{tabular}

Abbreviations: c-Kit, stem cell factor receptor; FGFR, fibroblast growth factor receptor; IC50, half-maximal inhibitory concentration; ND, not determined; PDGFR-B, platelet-derived growth factor receptor, beta; TKI, tyrosine kinase inhibitors; VEGFR, vascular endothelial growth factor. 
majority of patients showed secondary peaks, indicating that tivozanib may undergo enterohepatic recirculation, which would likely be a contributing factor in the observed long $t_{1 / 2}$.

The first-in-human Phase I and pharmacologic study of tivozanib was conducted in 41 patients with advanced solid tumors, ${ }^{47}$ including nine subjects with RCC. Tivozanib was administered at dose levels of $1.0 \mathrm{mg}, 1.5 \mathrm{mg}$, and $2.0 \mathrm{mg}$ per day for 28 days, followed by a 14-day break. The initial $2 \mathrm{mg}$ dose produced dose-limiting toxicity, with grade 3 proteinuria, hypertension, and ataxia. At the $1.5 \mathrm{mg}$ dose level of tivozanib, hypertension was the most common adverse event (62\% grade 3, $0 \%$ grade 4 ). Among the other grade 1 or 2 adverse events, dysphonia was seen in $56 \%$ and diarrhea in $31 \%$ of patients. Laboratory abnormalities included a low percentage of grade 3/4 liver function abnormalities and the absence of proteinuria. This study suggested a similar side effect spectrum to other oral VEGFR inhibitors. In another Phase II study, investigators administered tivozanib $1.5 \mathrm{mg} /$ day with a dosing schedule of 3 weeks on, 1 week off. Pharmacokinetic analysis and toxicity assessment was performed, and the results were consistent with those from previous tivozanib Phase I pharmacokinetic data in patients with advanced solid tumors and in healthy patients with no excess toxicity or drug accumulation. ${ }^{16,48,49}$ On that basis, the schedule of tivozanib $1.5 \mathrm{mg}$ daily for 3 weeks followed by a 1 -week break was adopted and used in all studies since.

Plasma levels of VEGF-A and soluble VEGFR-2 showed dose-dependent increases and decreases, respectively. Partial response was observed in two of nine patients with RCC. Six patients had stable disease and one patient disease progression. ${ }^{47}$ Subsequent evaluation of pharmacokinetic and toxicity in early studies suggested that a dosing regimen of 3 weeks on then 1 week off might be more optimal than 4 weeks on and 2 weeks off. Hence, this schedule was explored in the subsequent Phase II study.

\section{Phase II study}

The first Phase II trial of tivozanib enrolled 272 patients with a random discontinuation design for patients with stable disease; $83 \%$ had RCC with a clear cell component, $73 \%$ had undergone nephrectomy, and $46 \%$ had received prior therapy. Tivozanib was administered at a dose of $1.5 \mathrm{mg}$ daily for 3 weeks, followed by a 1 -week rest (3/1 week schedule). Median treatment duration was 8.5 months, while median PFS was 11.7 months (95\% confidence interval [CI], 8.3 to 14.3 months) with an overall response rate of $24 \%$ (95\% CI, 19\%-30\%). Subgroup analysis was also conducted to explore the effect of prior therapy, but there was no significant difference in PFS between patients who had had received prior therapy and those who were treatment naïve. The adverse events related to tivozanib were similar to those observed in the Phase I trial: hypertension (45\%) and dysphonia $(21 \%)$. $^{16,48,50}$

In the randomized discontinuation trial phase of the trial, 118 patients with stable disease were randomized to either tivozanib or a placebo. After 16 weeks of initial therapy, patients were allocated to further therapy based on the response. Patients with more than $25 \%$ tumor regression were continued on tivozanib, while patients with more than $25 \%$ tumor progression stopped therapy. Those patients judged to have stable disease were then randomized in a double-blind fashion to continue tivozanib or placebo for 12 weeks. In this group, the median PFS was longer for patients continuing tivozanib ( $\mathrm{n}=61 ; 10.3$ months) compared to the placebo group ( $\mathrm{n}=57 ; 3.3$ months). The data for all patients entered is summarized in Tables 2 and 3.

In summary, the results of this trial showed that tivozanib was active and well tolerated in patients with advanced RCC. The adverse event profile was acceptable and similar to those seen with other VEGFR inhibitors, including hypertension. ${ }^{16}$

\section{Phase III trials}

In TIVO-1, a Phase III randomized, open-label, multicenter trial, 517 patients with clear cell advanced RCC, with prior nephrectomy and Eastern Cooperative Oncology Group performance status 0 or 1 , were enrolled. Patients were treatment naïve or received no more than one prior systemic therapy for metastatic disease; those who were previously treated with VEGF- or mTOR-targeted therapy were excluded. Patients were randomized $1: 1$ to tivozanib $1.5 \mathrm{mg}$

Table 2 Overall radiologic response to tivozanib among patient subgroups

\begin{tabular}{|c|c|c|c|c|}
\hline $\begin{array}{l}\text { Patient } \\
\text { group }\end{array}$ & $\begin{array}{l}\text { Patient } \\
\text { number }\end{array}$ & $\begin{array}{l}\text { Median PFS } \\
\text { (months) }\end{array}$ & ORR (\%) & $\begin{array}{l}\begin{array}{l}\text { Duration } \\
\text { of response } \\
\text { (months) }\end{array} \\
\end{array}$ \\
\hline All patients & 272 & $\begin{array}{l}\text { II.7 } \\
(95 \% \mathrm{Cl}, \\
8.3-\mid 4.3)\end{array}$ & $\begin{array}{l}24 \\
(95 \% \mathrm{Cl}, \\
19-30)\end{array}$ & $\begin{array}{l}16.1 \\
(95 \% \mathrm{Cl}, \\
9.3-19.6)\end{array}$ \\
\hline Clear cell RCC & 226 & $\begin{array}{l}12.5 \\
(95 \% \mathrm{Cl}, \\
9.0-17.7)\end{array}$ & $\begin{array}{l}26 \\
(95 \% \mathrm{Cl} \text {, } \\
19-30)\end{array}$ & $\begin{array}{l}17.8 \\
(95 \% \mathrm{Cl}, \\
|2.0-2| .0)\end{array}$ \\
\hline $\begin{array}{l}\text { Clear cell RCC } \\
\text { and prior } \\
\text { nephrectomy }\end{array}$ & 176 & $\begin{array}{l}14.8 \\
(95 \% \mathrm{Cl} \\
10.3-19.2)\end{array}$ & $\begin{array}{l}30 \\
(95 \% \mathrm{Cl}, \\
23-37)\end{array}$ & $\begin{array}{l}16.1 \\
(95 \% \mathrm{Cl}, \\
11.2-19.6)\end{array}$ \\
\hline
\end{tabular}

Abbreviations: $\mathrm{Cl}$, confidence interval; ORR, objective response rate; PFS, progression-free survival; RCC, renal cell carcinoma. 
Table 3 Adverse events and laboratory abnormalities

\begin{tabular}{llll}
\hline Adverse event & All grades (\%) & Grade I/2 (\%) & Grade 3/4 (\%) \\
\hline Hypertension & 45 & 31 & 12 \\
Dysphonia & 22 & 22 & 0 \\
Asthenia & 10 & 7 & 3 \\
Diarrhea & 12 & 10 & 2 \\
Fatigue & 8 & 6 & 2 \\
Hand-foot & 4 & 4 & $<1$ \\
syndrome & & & \\
Stomatitis & 4 & 6 & $<1$ \\
ALT elevation & 29 & 28 & 1 \\
AST elevation & 27 & 26 & 1 \\
Direct bilirubin & 24 & 22 & 2
\end{tabular}

Abbreviations: ALT, alanine aminotransferase; AST, aspartate aminotransferase.

once daily for 3 weeks on, 1 week off, or to sorafenib $400 \mathrm{mg}$ twice daily continuously. Patients with protocol-defined progression on sorafenib were provided with tivozanib on an extension protocol; the design did not call for crossover from tivozanib to sorafenib as progression. Median PFS was 11.9 months for tivozanib, compared to 9.1 months for sorafenib (hazard ratio [HR], 0.797; 95\% CI, 0.639-0.993; $P=0.042$ ). In the treatment-naïve subgroup, the median PFS was 12.7 months for tivozanib and 9.1 months for sorafenib (HR, 0.756; 95\% CI, 0.580-0.985; $P=0.037$ ). OS trended in favor of sorafenib (stratified HR, 1.245; 95\% CI, 0.954-1.624; $P=0.105$ ). Tivozanib demonstrated significant improvement in PFS and ORR compared with sorafenib as initial targeted treatment for advanced RCC. The safety profile of tivozanib was favorable, with significantly less hand-foot syndrome ( $13 \%$ versus $54 \%$ ), diarrhea ( $22 \%$ versus $32 \%$ ), and alopecia ( $2 \%$ versus $21 \%$ ) compared to sorafenib. Hypertension (44\% versus 34\%), back pain ( $14 \%$ versus $7 \%)$, and dysphonia ( $21 \%$ versus $5 \%$ ) were significantly more common in the tivozanib arm. ${ }^{15}$

In the extension study, patients with progressive disease (PD) on sorafenib and patients with PD on tivozanib received subsequent treatment. Of the 257 patients on sorafenib, 155 $(60.3 \%)$ were treated with next-line tivozanib at the time of the analysis. At the time of final OS analysis, which was 2 years after the last patient was enrolled, 118 deaths had occurred in the tivozanib arm (45.4\%) compared to the sorafenib arm, where 101 deaths occurred (39.3\%). There was no significant difference in OS between the two treatment arms (28.8 months for tivozanib versus 29.3 months for sorafenib). After discontinuation of initial therapy, $64 \%$ of patients in the tivozanib arm received no next-line therapy, compared with $26 \%$ of patients in the control arm. The authors suggested that the high rate of utilization of secondline tivozanib in patients following PD on sorafenib might have affected the OS outcome. ${ }^{51}$ The key additional factor in this trial was that patients, many of whom resided in Eastern Europe, initially received tivozanib but had limited access to additional targeted therapies with efficacy in metastatic RCC. ${ }^{15}$ This meant that those initially given sorafenib received two drugs, compared to the one drug administered to those given tivozanib.

In the Phase II extension study of tivozanib for patients crossing over from sorafenib, RCC patients who were treated with tivozanib after progression on sorafenib were evaluated. Partial response (PR) was 7.9\%; stable disease (SD) was $65.4 \%$, and $71.3 \%$ of patients showed some degree of tumor shrinkage. Median duration of PR, SD, and PFS was 11.1 months (95\% CI, 7.5-not reported [NR] months), 12.7 months (95\% CI, 7.4-NR months), and 5.6 months (95\% CI, 5.4-9.1 months), respectively. The preliminary data demonstrated that tivozanib has antitumor activity after PD on sorafenib. The adverse event profile of tivozanib after sorafenib was similar to that observed in TIVO- $1 .^{52}$

The subgroup analysis from TIVO-1 showed significant improvement in PFS by tivozanib compared to sorafenib. The PFS advantage was observed in those patients who were of Caucasian descent, had an Eastern Cooperative Oncology Group performance status of 0 , who were diagnosed more than 1 year prior, who had received no prior treatment, who had more than 2 metastatic sites, whose baseline systolic blood pressure (BP) was greater than $140 \mathrm{mmHg}$, and whose baseline diastolic BP was less than $90 \mathrm{mmHg}$. Patients who developed hypertension during the study had significantly longer PFS than patients with normal BP. The improvement in PFS was more marked for tivozanib compared to sorafenib for the patients who developed hypertension. ${ }^{53}$ Similar results were previously reported by Rini et $\mathrm{al}^{54}$ in patients with metastatic RCC, whereby sunitinib-associated HTN is associated with improved clinical outcomes.

Health-related quality of life assessment was generally similar for tivozanib and sorafenib. The physical well-being assessment demonstrated more significant improvement with tivozanib compared to sorafenib. ${ }^{55}$ The data for Phase III trials utilizing VEGF inhibitors or VEGF antibodies in advanced RCC are summarized in Tables 4 and 5.

\section{Future aspects}

Tivozanib will be compared to sunitinib in the TAURUS trial, a first-line randomized Phase II patient preference trial in metastatic RCC. In this trial design (modeled on the PISCES trial, which compared pazopanib and sunitinib), patients are randomized to either tivozanib or sunitinib for 
Table 4 Phase III trials utilizing VEGF inhibitors or VEGF antibodies in advanced RCC survival and toxicity data

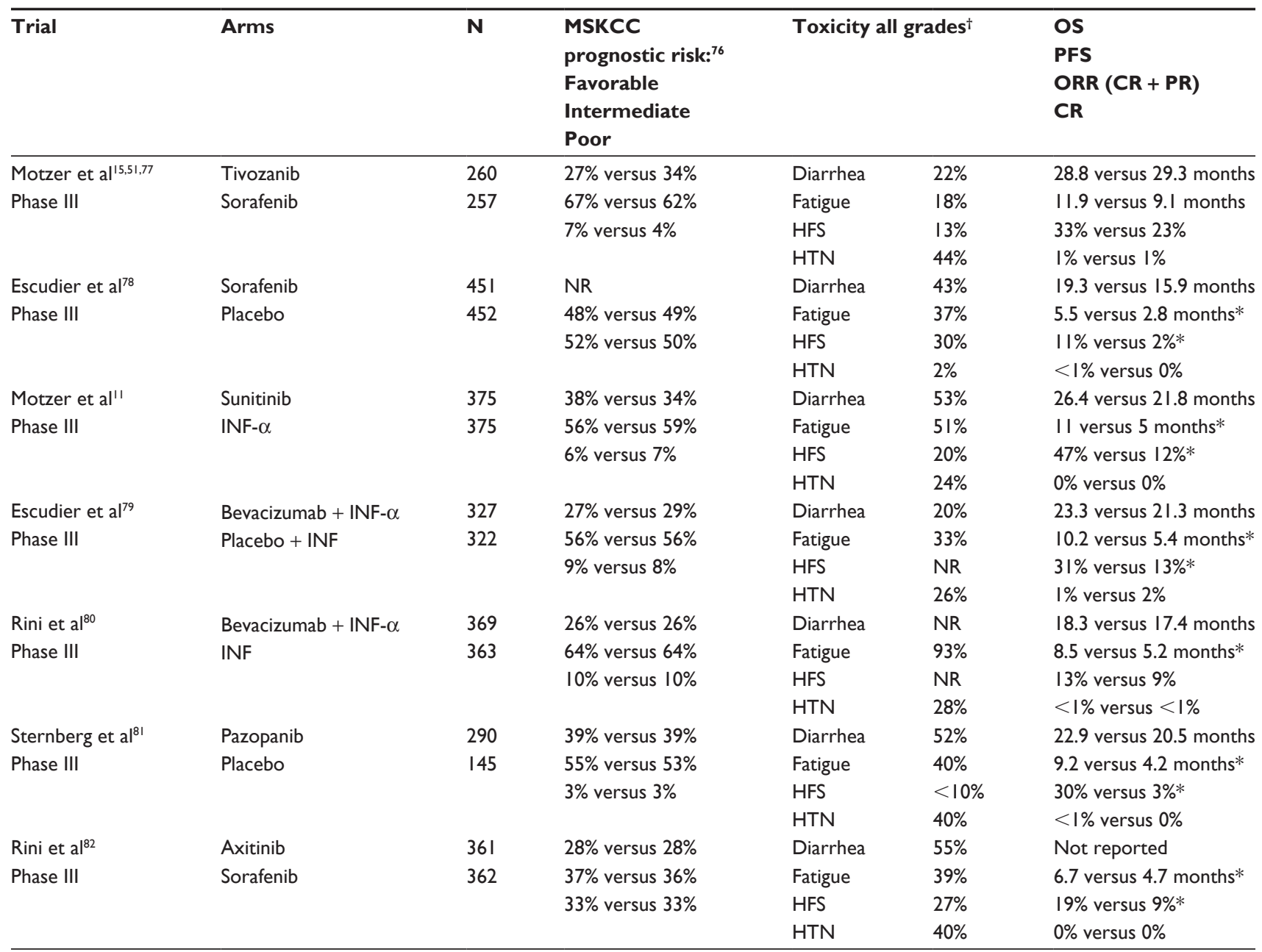

Notes: *Not significant; ${ }^{\dagger}$ toxicity for agent in the intervention arm.

Abbreviations: CR, complete response; HFS, hand-foot syndrome; HTN, hypertension; INF, interferon; MSKCC, Memorial Sloan-Kettering Cancer Center; N, number; NR, not reported; ORR, objective response rate; OS, overall survival; PFS, progression-free survival; PR, partial response; RCC, renal cell carcinoma; VEGF, vascular endothelial growth factor.

12 weeks, followed by a restaging and toxicity assessment and crossover to the other drug for 12 weeks. Patients are then reassessed and state a preference for either drug.

\section{Combination therapies in RCC}

Currently approved targeted therapy improved PFS and OS in advanced RCC; however, durable responses are infrequent, and resistance is commonly seen after a median of 6-15 months. ${ }^{56}$ Despite maximal inhibition of VEGF/VEGFR and PDGF/PDGFR pathways, resistance reliably develops due to a process known as "reactive resistance." There are two major models that explain the resistance to VEGF inhibitors, which are adaptive and intrinsic. The adaptive mechanism of resistance hypothesizes that tumors elude the VEGF inhibitor effects and salvage their functionality through an escape mechanism. ${ }^{57}$ In preclinical models, HIF1 alpha and HIF2alpha are major mediators of this process. ${ }^{58,59}$ In a xenograft model, it was shown that increased IL-8 was consistent with escape from antiangiogenic agents. Administration of IL-8 neutralizing antibody successfully sensitized tumors to sunitinib, which supported this hypothesis. ${ }^{60}$ Increased placental growth factor levels, angiopoietin pathway, and PDGFR have been associated with emergence of alternative proangiogenic mechanisms that would lead to resistance. ${ }^{61-64}$ Intrinsic resistance defines the types of tumors that would not respond to VEGF inhibitors under any circumstances. This mechanism has been only shown in animal models. ${ }^{65}$ Tumors that are driven by this pathway develop progressive disease soon after VEGF inhibitor treatment has been started. However, given that pathways other than VEGF or mTOR play a role in tumorigenesis, ${ }^{57}$ other pathways may also be related to resistance to VEGF or mTOR-targeted therapy in RCC. For instance, basic fibroblast growth factor, hepatocyte growth factor, and IL-6 have recently been shown to increase 
Table 5 Phase III trials utilizing VEGF inhibitors or VEGF antibodies in advanced RCC discontinuation data

\begin{tabular}{|c|c|c|c|c|c|c|c|c|}
\hline \multirow[t]{3}{*}{ Trial } & \multirow[t]{3}{*}{ Arms } & \multirow[t]{3}{*}{$\mathbf{N}$} & \multicolumn{6}{|c|}{ Reasons to discontinue therapy } \\
\hline & & & \multicolumn{2}{|c|}{ PD or death } & \multicolumn{2}{|c|}{ Toxicity } & \multicolumn{2}{|c|}{ Patient choice } \\
\hline & & & $\mathbf{N}$ & $\%$ & $\mathbf{N}$ & $\%$ & $\mathbf{N}$ & $\%$ \\
\hline Motzer et al $\left.\right|^{15,77}$ & Tivozanib & 260 & 189 & 72 & 4 & 1 & NR & NR \\
\hline Phase III & Sorafenib & 257 & 226 & 87 & 5 & 2 & NR & NR \\
\hline Escudier et $\mathrm{al}^{78}$ & Sorafenib & 451 & 246 & 55 & 18 & 4 & 7 & 2 \\
\hline Phase III & Placebo & 452 & 300 & 66 & 17 & 4 & 11 & 2 \\
\hline Motzer et al" & Sunitinib & 375 & 92 & 25 & 30 & 8 & 4 & I \\
\hline Phase III & INF- $\alpha$ & 375 & 170 & 45 & 47 & 13 & 16 & 4 \\
\hline Escudier et $\mathrm{al}^{79}$ & Bevacizumab + INF- $\alpha$ & 327 & $|5|$ & 46 & 86 & 26 & 10 & 10 \\
\hline Phase III & Placebo + INF- $\alpha$ & 322 & 224 & 70 & 32 & 10 & 11 & 11 \\
\hline Rini et $\mathrm{a}^{80}$ & Bevacizumab + INF- $\alpha$ & 369 & 200 & 54 & 85 & 23 & 40 & 11 \\
\hline Phase III & INF- $\alpha$ & 363 & 218 & 60 & 66 & 18 & 33 & 3 \\
\hline Sternberg et $\mathrm{a}^{81}$ & Pazopanib & 290 & 158 & 54 & 41 & 14 & 18 & 6 \\
\hline Phase III & Placebo & 145 & 121 & 83 & 5 & 3 & 4 & 4 \\
\hline Rini et $\mathrm{al}^{82}$ & Axitinib & 361 & 172 & 48 & 22 & 6 & 10 & 3 \\
\hline Phase III & Sorafenib & 362 & 193 & 53 & 33 & 9 & 7 & 2 \\
\hline
\end{tabular}

Abbreviations: INF, interferon; N, number; NR, not reported; PD, progressive disease; RCC, renal cell carcinoma; VEGF, vascular endothelial growth factor.

or remain high immediately before progression in patients being treated with sunitinib. ${ }^{66}$

Because the activation of multiple parallel pathways leads to VEGF resistance, investigators have postulated that adding inhibitors of other pathways might mitigate resistance and improve outcome.

Given significant activity in RCC, mTOR inhibitors and VEGF inhibitors are an obvious potential combination to provide targeted inhibition tumor cell growth via parallel and overlapping pathways in the context of different side effect profiles. ${ }^{41}$ Previously tested combinations of mTOR inhibitors with sunitinib or sorafenib were either not tolerated or tolerated at attenuated doses due to adverse event profile. ${ }^{67,68}$ More recently, the TORAVA randomized Phase II trial assessing the safety profile and efficacy of bevacizumab and temsirolimus demonstrated a worse toxicity profile and poorer disease control than sunitinib. ${ }^{69}$ In contrast, a Phase I study of the combination of everolimus and sorafenib at submaximal doses produced acceptable toxicity and evidence of antitumor activity in previously untreated patients with metastatic RCC. ${ }^{70}$

Tivozanib was combined with temsirolimus in a Phase IB study. In contrast to other combinations, the authors reported that full doses of tivozanib $(1.5 \mathrm{mg} /$ day $)$ and temsirolimus (25 mg/week) were tolerable, with no dose-limiting toxicities. Activity in terms of prolonged stable disease and partial response was reported in subjects on the trial. These results are promising, since tivozanib was the first selective VEGFR TKI to be successfully combined with an mTOR inhibitor at the full recommended dose and schedule of both agents. ${ }^{71,72}$
A recent study has demonstrated activity of tivozanib after treatment with VEGF inhibitors, specifically sorafenib. ${ }^{52}$ Additional studies are warranted to determine whether tivozanib, alone or in combination, may be effective after resistance to other VEGF-targeted drugs develops.

\section{Nonkidney cancer clinical development directions with tivozanib}

Tivozanib is also currently being evaluated in patients with other cancer types and demonstrated promising results. Phase I studies showed that tivozanib can be safely combined at full doses and schedule with paclitaxel in metastatic breast cancer and FOLFOX6 in advanced gastrointestinal tumors. ${ }^{49,73}$ A study evaluating the combination of tivozanib with everolimus in metastatic colorectal cancer is ongoing (ClinicalTrials.gov identifier: NCT01058655). In another open-label trial, tivozanib is being investigated in patients with nonsmall cell lung cancer (ClinicalTrials.gov identifier: NCT00826878).

\section{Biomarkers associated with tivozanib response}

Tumor-associated macrophages enhance immunosuppression and angiogenesis by secreting VEGF, cytokines, and other angiogenesis factors. These tumor-associated macrophages are regulated by myeloid-derived suppressor cells. Myeloid-derived suppressor cells and their secreted alternative ligands are associated in the development of resistance to bevacizumab and VEGF receptor TKI therapy. ${ }^{40}$ 
Myeloid cells have also been investigated as a predictor of tivozanib efficacy. In a preclinical study to identify biomarkers associated with tivozanib response, a population-based tumor model comprising more than 100 genetically developed breast HER2 tumors was used to test the efficacy of tivozanib. IHC analysis of infiltrating myeloid cells in 21 patient samples demonstrated a significant correlation between the percent myeloid cell composition in the tumors and maximum tumor shrinkage by RECIST (Response Evaluation Criteria In Solid Tumors) criteria. The data demonstrated the presence of tivozanib sensitive and insensitive angiogenesis mechanisms in both murine and human solid tumors. These findings provided evidence for a potential biomarker that could be utilized to predict tivozanib response. ${ }^{36}$

The Phase II BATON trial was designed to further validate a tivozanib-resistance biomarker, as well as to determine the biomarkers that may predict clinical activity and/or toxicity with tivozanib. Investigators identified a 42-gene resistance signature defining a specific tumor infiltrating myeloid population by using a novel, coherence-based bioinformatics analysis of pretreatment tumor microarray data. They analyzed 21 samples from patients treated with tivozanib in a Phase II RCC trial. The data demonstrated that the percent of myeloid cell composition in the tumors and clinical antitumor activity of tivozanib was significantly correlated (ClinicalTrial.gov identifier: NCT01297244). ${ }^{74}$

In a more recent study, investigators characterized molecularly annotated datasets that were available from a Phase III TIVO-1 trial to further characterize molecular RCC subtypes and evaluate relationships between subtypes and VEGF TKI activity. Based on their analysis, the hypoxia signature was significantly associated with better PFS on tivozanib. The study demonstrated a distinct molecular profile, which can be classified by a low hypoxia signature and may help identify tivozanib responders. ${ }^{75}$

\section{Conclusion}

In conclusion, tivozanib is a novel TKI with relative selectivity for VEGF receptors compared to earlier drugs. Tivozanib demonstrated better response rates and PFS in a Phase III study in a composite group of patients who were therapy naïve or who had had one line of therapy, typically with cytokines. The safety profile of tivozanib differed from sorafenib with less hand-foot skin reactions and diarrhea, and more hypertension. Phase I data suggests that tivozanib and temsirolimus can be given together in full therapeutic doses, while other trials investigating combinations are ongoing. The TAURUS clinical trial is currently investigating the efficacy of tivozanib versus sunitinib in a first-line setting (ClinicalTrials.gov identifier: NCT01673386). However, given the target and toxicity profile of tivozanib, the setting in which this agent may be used to greatest advantage in the current RCC landscape is in first-line therapy for metastatic disease. A first-line Phase III trial of tivozanib compared to pazopanib is in its planning phase. Tivozanib will also be explored in other cancers including colorectal, lung, breast, and hepatobiliary cancers.

\section{Disclosure}

Tanya B Dorff is a consultant for Novartis, and does promotional speaking for Bayer and Pfizer. David I Quinn is a consultant for Pfizer, Bayer, Novartis, Aveo, and Prometheus. The other authors report no conflicts of interest in this work.

\section{References}

1. Siegel R, Naishadham D, Jemal A. Cancer statistics, 2012. CA Cancer J Clin. 2012;62(1):10-29.

2. Howlader N, Ries LA, Mariotto AB, Reichman ME, Ruhl J, Cronin KA. Improved estimates of cancer-specific survival rates from populationbased data. J Natl Cancer Inst. 2010;102(20):1584-1598.

3. Sakamoto S, Ryan AJ, Kyprianou N. Targeting vasculature in urologic tumors: mechanistic and therapeutic significance. J Cell Biochem. 2008;103(3):691-708.

4. Ljungberg B, Hanbury DC, Kuczyk MA, et al; for European Association of Urology Guideline Group for renal cell carcinoma. Renal cell carcinoma guideline. Eur Urol. 2007;51(6):1502-1510.

5. Motzer RJ, Bander NH, Nanus DM. Renal-cell carcinoma. N Engl J Med. 1996;335(12):865-875.

6. Negrier S, Escudier B, Lasset C, et al. Recombinant human interleukin-2, recombinant human interferon alfa-2a, or both in metastatic renal-cell carcinoma. Groupe Français d'Immunothérapie. N Engl J Med. 1998; 338(18):1272-1278.

7. Motzer RJ, Mazumdar M, Bacik J, Russo P, Berg WJ, Metz EM. Effect of cytokine therapy on survival for patients with advanced renal cell carcinoma. J Clin Oncol. 2000;18(9):1928-1935.

8. Kapoor AK, Hotte SJ. Current status of cytokine therapy in management of patients with metastatic renal cell carcinoma. Can Urol Assoc J. 2007;1(Supp1 2):S28-S33.

9. Motzer RJ, Bacik J, Murphy BA, Russo P, Mazumdar M. Interferon-alfa as a comparative treatment for clinical trials of new therapies against advanced renal cell carcinoma. J Clin Oncol. 2002;20(1):289-296.

10. Pal SK, Williams S, Josephson DY, Carmichael C, Vogelzang NJ, Quinn DI. Novel therapies for metastatic renal cell carcinoma: efforts to expand beyond the VEGF/mTOR signaling paradigm. Mol Cancer Ther. 2012;11(3):526-537.

11. Motzer RJ, Hutson TE, Tomczak P, et al. Sunitinib versus interferon alfa in metastatic renal-cell carcinoma. $N$ Engl J Med. 2007;356(2): 115-124.

12. Motzer RJ, Hutson TE, Tomczak P, et al. Overall survival and updated results for sunitinib compared with interferon alfa in patients with metastatic renal cell carcinoma. J Clin Oncol. 2009;27(22): 3584-3590.

13. Hudes G, Carducci M, Tomczak P, et al. Temsirolimus, interferon alfa, or both for advanced renal-cell carcinoma. N Engl J Med. 2007; 356(22):2271-2281.

14. Coppin C, Kollmannsberger C, Le L, Porzsolt F, Wilt TJ. Targeted therapy for advanced renal cell cancer (RCC): a Cochrane systematic review of published randomised trials. BJU Int. 2011;108(10):1556-1563. 
15. Motzer RJ, Nosov D, Eisen T, et al. Tivozanib versus sorafenib as initial targeted therapy for patients with advanced renal cell carcinoma: Results from a phase III randomized, open-label, multicenter trial [abstract]. $J$ Clin Oncol. 2012;30:277s.

16. Nosov DA, Esteves B, Lipatov ON, et al. Antitumor activity and safety of tivozanib (AV-951) in a phase II randomized discontinuation trial in patients with renal cell carcinoma. J Clin Oncol. 2012;30(14):1678-1685.

17. Clifford SC, Prowse AH, Affara NA, Buys CH, Maher ER. Inactivation of the von Hippel-Lindau (VHL) tumour suppressor gene and allelic losses at chromosome arm $3 \mathrm{p}$ in primary renal cell carcinoma: evidence for a VHL-independent pathway in clear cell renal tumourigenesis. Genes Chromosomes Cancer. 1998;22(3):200-209.

18. Rafii S, Lyden D, Benezra R, Hattori K, Heissig B. Vascular and haematopoietic stem cells: novel targets for anti-angiogenesis therapy? Nat Rev Cancer. 2002;2(11):826-835.

19. Herman JG, Latif F, Weng Y, et al. Silencing of the VHL tumorsuppressor gene by DNA methylation in renal carcinoma. Proc Natl Acad Sci U S A. 1994;91(21):9700-9704.

20. Kondo K, Kaelin WG Jr. The von Hippel-Lindau tumor suppressor gene. Exp Cell Res. 2001;264(1):117-125.

21. Kondo K, Yao M, Yoshida M, et al. Comprehensive mutational analysis of the VHL gene in sporadic renal cell carcinoma: relationship to clinicopathological parameters. Genes Chromosomes Cancer. 2002 34(1):58-68.

22. Kondo Y, Shen L, Issa JP. Critical role of histone methylation in tumor suppressor gene silencing in colorectal cancer. Mol Cell Biol. 2003; 23(1):206-215.

23. Youssef EM, Chen XQ, Higuchi E, et al. Hypermethylation and silencing of the putative tumor suppressor Tazarotene-induced gene 1 in human cancers. Cancer Res. 2004;64(7):2411-2417.

24. Banks RE, Tirukonda $\mathrm{P}$, Taylor $\mathrm{C}$, et al. Genetic and epigenetic analysis of von Hippel-Lindau (VHL) gene alterations and relationship with clinical variables in sporadic renal cancer. Cancer Res. 2006;66(4): 2000-2011.

25. Kucejova B, Peña-Llopis S, Yamasaki T, et al. Interplay between pVHL and mTORC1 pathways in clear-cell renal cell carcinoma. Mol Cancer Res. 2011;9(9):1255-1265.

26. Haddad H, Rini BI. Current treatment considerations in metastatic renal cell carcinoma. Curr Treat Options Oncol. 2012;13(2): 212-229.

27. Ducker GS, Atreya CE, Simko JP, et al. Incomplete inhibition of phosphorylation of 4E-BP1 as a mechanism of primary resistance to ATP-competitive mTOR inhibitors. Oncogene. Epub April 1, 2013.

28. Sulzbacher I, Birner P, Träxler M, Marberger M, Haitel A. Expression of platelet-derived growth factor-alpha alpha receptor is associated with tumor progression in clear cell renal cell carcinoma. Am J Clin Pathol. 2003;120(1):107-112.

29. Rini BI, Small EJ. Biology and clinical development of vascular endothelial growth factor-targeted therapy in renal cell carcinoma J Clin Oncol. 2005;23(5):1028-1043.

30. Ferrara N. Vascular endothelial growth factor: basic science and clinical progress. Endocr Rev. 2004;25(4):581-611.

31. Leung DW, Cachianes G, Kuang WJ, Goeddel DV, Ferrara N. Vascular endothelial growth factor is a secreted angiogenic mitogen. Science. 1989;246(4935):1306-1309.

32. George DJ, Kaelin WG Jr. The von Hippel-Lindau protein, vascular endothelial growth factor, and kidney cancer. $N$ Engl J Med. 2003; 349(5):419-421

33. Kowanetz M, Ferrara N. Vascular endothelial growth factor signaling pathways: therapeutic perspective. Clin Cancer Res. 2006;12(17) 5018-5022.

34. Kusmartsev S, Su Z, Heiser A, et al. Reversal of myeloid cell-mediated immunosuppression in patients with metastatic renal cell carcinoma. Clin Cancer Res. 2008;14(24):8270-8278.

35. Ko JS, Rayman P, Ireland J, et al. Direct and differential suppression of myeloid-derived suppressor cell subsets by sunitinib is compartmentally constrained. Cancer Res. 2010;70(9):3526-3536.
36. Lin J, Sun X, Feng B, et al. Tivozanib biomarker identifies tumor infiltrating myeloid cells contributing to tivozanib resistance in both preclinical models and human renal cell carcinoma [abstract]. EJC Supplements. 2010;8:191.

37. McDermott DF. Immunotherapy of metastatic renal cell carcinoma. Cancer. 2009;115(Suppl 10):S2298-S2305.

38. Topalian SL, Hodi FS, Brahmer JR, et al. Safety, activity, and immune correlates of anti-PD-1 antibody in cancer. NEngl J Med. 2012;366(26): 2443-2454.

39. Brahmer JR, Tykodi SS, Chow LQ, et al. Safety and activity of antiPD-L1 antibody in patients with advanced cancer. $N$ Engl J Med. 2012;366(26):2455-2465.

40. Biswas S, Eisen T. Immunotherapeutic strategies in kidney cancer when TKIs are not enough. Nat Rev Clin Oncol. 2009;6(8):478-487.

41. Mulders P. Vascular endothelial growth factor and mTOR pathways in renal cell carcinoma: differences and synergies of two targeted mechanisms. BJU Int. 2009;104(11):1585-1589.

42. Shibuya M. Vascular endothelial growth factor receptor-2: its unique signaling and specific ligand, VEGF-E. Cancer Sci. 2003;94(9): 751-756.

43. Nakamura K, Taguchi E, Miura T, et al. KRN951, a highly potent inhibitor of vascular endothelial growth factor receptor tyrosine kinases, has antitumor activities and affects functional vascular properties. Cancer Res. 2006;66(18):9134-9142.

44. Taguchi E, Nakamura K, Miura T, Shibuya M, Isoe T. Anti-tumor activity and tumor vessel normalization by the vascular endothelial growth factor receptor tyrosine kinase inhibitor KRN951 in a rat peritoneal disseminated tumor model. Cancer Sci. 2008;99(3):623-630.

45. Pal SK, Bergerot PG, Figlin RA. Tivozanib: current status and future directions in the treatment of solid tumors. Expert Opin Investig Drugs. 2012;21(12):1851-1859.

46. Cotreau MM, Hale CL, Jacobson L, et al. Absorption, metabolism, and excretion of [14c]-tivozanib, a vascular endothelial growth factor receptor tyrosine kinase inhibitor, in healthy male participants: a phase I, open-label, mass-balance study. Clinical Pharmacology in Drug Development. 2012;1:102-109.

47. Eskens FA, de Jonge MJ, Bhargava P, et al. Biologic and clinical activity of tivozanib (AV-951, KRN-951), a selective inhibitor of VEGF receptor-1, -2, and -3 tyrosine kinases, in a 4-week-on, 2-week-off schedule in patients with advanced solid tumors. Clin Cancer Res. 2011;17(22):7156-7163.

48. Eskens F, Oldenhuis CN, Bhargava P, et al. A phase Ib, open-label, dose-escalation study of tivozanib and FOLFOX6 in patients (pts) with advanced gastrointestinal (GI) tumors [abstract]. J Clin Oncol. 2011;29:549.

49. Eskens F, Oldenhuis CN, Loos WJ, et al. Final results of a phase Ib study of tivozanib and FOLFOX6 in patients (pts) with advanced gastrointestinal (GI) tumors [abstract]. J Clin Oncol. 2012;30:4132.

50. Bhargava P, Esteves B, Al-Adhami M, et al. Activity of tivozanib (AV951) in patients with renal cell carcinoma (RCC): Subgroup analysis from a phase II randomized discontinuation trial (RDT) [abstract]. $J$ Clin Oncol. 2010;28:4599.

51. Motzer RJ, Eisen T, Hutson TE, et al. Overall survival results from a phase III study of tivozanib hydrochloride versus sorafenib in patients with renal cell carcinoma [abstract]. J Clin Oncol. 2013; 31(Suppl 6):350.

52. Motzer RJ, Nosov D, Tomczak P, et al. Efficacy and safety data from patients with advanced renal cell cancer treated with tivozanib hydrochloride after progression on sorafenib [abstract]. J Clin Oncol. 2013;31(Suppl 6):364

53. Hutson TE, Nosov D, Eisen T, et al. Subgroup analyses of a phase III trial comparing tivozanib hydrochloride versus sorafenib as initial targeted therapy for patients (pts) with metastatic renal cell carcinoma (mRCC) [abstract]. J Clin Oncol. 2013;31(Suppl 6):354.

54. Rini BI, Cohen DP, Lu DR, et al. Hypertension as a biomarker of efficacy in patients with metastatic renal cell carcinoma treated with sunitinib. J Natl Cancer Inst. 2011;103(9):763-773. 
55. Cella D, Ivanescu C, Skaltsa K, et al. Treatment benefit of tivozanib hydrochloride versus sorafenib on health-related quality of life (HRQoL) among patients (pts) with advanced/metastatic renal cell carcinoma (mRCC): TIVO-1study results [abstract]. J Clin Oncol. 2013;31:355.

56. Santos N, Wenger JB, Havre P, et al. Combination therapy for renal cell cancer: what are possible options? Oncology. 2011;81(3-4):220-229.

57. Sadeghi S, Garcia JA. Overcoming VEGF resistance in renal cancer: biologic and therapeutic implications. Clin Investig (Lond). 2012;2(6): 615-621.

58. Blagosklonny MV. Antiangiogenic therapy and tumor progression. Cancer Cell. 2004;5(1):13-17.

59. Casanovas O, Hicklin DJ, Bergers G, Hanahan D. Drug resistance by evasion of antiangiogenic targeting of VEGF signaling in late-stage pancreatic islet tumors. Cancer Cell. 2005;8(4):299-309.

60. Huang D, Ding Y, Zhou M, et al. Interleukin-8 mediates resistance to antiangiogenic agent sunitinib in renal cell carcinoma. Cancer Res. 2010;70(3):1063-1071.

61. Batchelor TT, Sorensen AG, di Tomaso E, et al. AZD2171, a pan-VEGF receptor tyrosine kinase inhibitor, normalizes tumor vasculature and alleviates edema in glioblastoma patients. Cancer Cell. 2007;11(1):83-95.

62. Rini B, Szczylik C, Tannir NM, et al. AMG 386 in combination with sorafenib in patients with metastatic clear cell carcinoma of the kidney: a randomized, double-blind, placebo-controlled, phase 2 study. Cancer. 2012;118(24):6152-6161.

63. Bergers G, Song S, Meyer-Morse N, Bergsland E, Hanahan D. Benefits of targeting both pericytes and endothelial cells in the tumor vasculature with kinase inhibitors. J Clin Invest. 2003;111(9):1287-1295.

64. Eisen T, Joensuu H, Nathan PD, et al. Regorafenib for patients with previously untreated metastatic or unresectable renal-cell carcinoma: a single-group phase 2 trial. Lancet Oncol. 2012;13(10):1055-1062.

65. Yao X, Qian CN, Zhang ZF, et al. Two distinct types of blood vessels in clear cell renal cell carcinoma have contrasting prognostic implications. Clin Cancer Res. 2007;13(1):161-169.

66. Porta C, Paglino C, Imarisio I, et al. Changes in circulating proangiogenic cytokines, other than VEGF, before progression to sunitinib therapy in advanced renal cell carcinoma patients. Oncology. 2013; 84(2):115-122.

67. Patel PH, Senico PL, Curiel RE, Motzer RJ. Phase I study combining treatment with temsirolimus and sunitinib malate in patients with advanced renal cell carcinoma. Clin Genitourin Cancer. 2009; 7(1):24-27.

68. Molina AM, Feldman DR, Voss MH, et al. Phase 1 trial of everolimus plus sunitinib in patients with metastatic renal cell carcinoma. Cancer. 2012;118(7):1868-1876.

69. Negrier S, Gravis G, Pérol D, et al. Temsirolimus and bevacizumab, or sunitinib, or interferon alfa and bevacizumab for patients with advanced renal cell carcinoma (TORAVA): a randomised phase 2 trial. Lancet Oncol. 2011;12(7):673-680.
70. Harzstark AL, Small EJ, Weinberg VK, et al. A phase 1 study of everolimus and sorafenib for metastatic clear cell renal cell carcinoma. Cancer. 2011;117(18):4194-4200.

71. Fishman MN, Srinivas SRJ, Hauke RJ, et al. Phase Ib study of tivozanib [AV-951] in combination with temsirolimus in patients with renal cell carcinoma. In press 2013.

72. Kabbinavar FF, Srinivas S, Hauke RJ, et al. A phase I trial of combined tivozanib (AV-951) and temsirolimus therapy in patients (pts) with renal cell carcinoma (RCC) [abstract]. J Clin Oncol. 2011; 29(Suppl 7):330.

73. Mayer EL, Scheulen ME, Beckman J, et al. Combination of tivozanib (AV-951) with weekly paclitaxel for metastatic breast cancer: Results of a phase I study [abstract]. J Clin Oncol. 2011;29:1092.

74. Hutson TE, Rathmell K, Hudes GR, et al. A phase II biomarker assessment of tivozanib in oncology (BATON) trial in patients (pts) with advanced renal cell carcinoma (RCC) [abstract]. J Clin Oncol. 2012;30:TPS4686.

75. Robinson MO, Feng B, Nicoletti R, et al. Relationship of hypoxia signature with variant subgroup of clear cell renal cell carcinoma (ccRCC) and its association with clinical activity on tivozanib hydrochloride [abstract]. J Clin Oncol. 2013;31(Suppl 6):361.

76. Motzer RJ, Bacik J, Mazumdar M. Prognostic factors for survival of patients with stage IV renal cell carcinoma: Memorial SloanKettering Cancer Center experience. Clin Cancer Res. 2004; 10(18 Pt 2):6302S-6303S.

77. Motzer RJ, Bhargava P, Esteves B, et al. A phase III, randomized, controlled study to compare tivozanib with sorafenib in patients (pts) with advanced renal cell carcinoma (RCC) [abstract]. J Clin Oncol. 2011;29(Suppl 7):310.

78. Escudier B, Eisen T, Stadler WM, et al. Sorafenib in advanced clear-cell renal-cell carcinoma. $N$ Engl J Med. 2007;356(2):125-134.

79. Escudier B, Pluzanska A, Koralewski P, et al; for AVOREN Trial investigators. Bevacizumab plus interferon alfa-2a for treatment of metastatic renal cell carcinoma: a randomised, double-blind phase III trial. Lancet. 2007;370(9605):2103-2111.

80. Rini BI, Halabi S, Rosenberg JE, et al. Bevacizumab plus interferon alfa compared with interferon alfa monotherapy in patients with metastatic renal cell carcinoma: CALGB 90206. J Clin Oncol. 2008;26(33): 5422-5428.

81. Sternberg CN, Davis ID, Mardiak J, et al. Pazopanib in locally advanced or metastatic renal cell carcinoma: results of a randomized phase III trial. J Clin Oncol. 2010;28(6):1061-1068.

82. Rini BI, Escudier B, Tomczak P, et al. Comparative effectiveness of axitinib versus sorafenib in advanced renal cell carcinoma (AXIS): a randomised phase 3 trial. Lancet. 2011;378(9807):1931-1939.

\section{Publish your work in this journal}

Biologics: Targets \& Therapy is an international, peer-reviewed journal focusing on the patho-physiological rationale for and clinical application of Biologic agents in the management of autoimmune diseases, cancers or other pathologies where a molecular target can be identified. This journal is indexed on PubMed Central, CAS, EMBase, Scopus

\section{Dovepress}

and the Elsevier Bibliographic databases. The manuscript management system is completely online and includes a very quick and fair peerreview system, which is all easy to use. Visit http://www.dovepress com/testimonials.php to read real quotes from published authors. 